\title{
A doublet mechanics model for the ultrasound characterization of malignant tissues
}

\author{
Francesco Gentile $^{1,2}$, Jason Sakamoto ${ }^{3}$, Raffaella Righetti ${ }^{4}$, Paolo Decuzzi ${ }^{1,3}$, Mauro Ferrari ${ }^{3,5}$ \\ ${ }^{1}$ Center of Bio-Nanotechnology and Engineering for Medicine, Magna Graecia University, Catanzaro, Italy; \\ ${ }^{2}$ Italian Institute of Technology, Genova, Italy; \\ ${ }^{3}$ Department of Nanomedicine and Biomedical Engineering, The Methodist Hospital Research Institute, Houston, USA; \\ ${ }^{4}$ Department of Electrical and Computer Engineering, Texas A\&M, 214 Zachry Engineering Center, College Station, USA; \\ ${ }^{5}$ Anderson Cancer Center and Rice University, Houston, USA. \\ Email: francesco.gentile@,iit.it
}

Received 30 October 2010; revised 4 November 2011; accepted 4 January 2011.

\begin{abstract}
Non invasive ultrasound-based imaging systems are being more commonly used in clinical bio-microscopy applications for both ex vivo and in vivo analysis of tissue pathological and physiological states. These modalities usually employ high-frequency ultrasound systems to overcome spatial resolution limits of conventional clinical diagnostic approaches. Biological tissues are non continuous, non homogeneous and exhibit a multiscale organization from the sub-cellular level $(\leq 1 \mathrm{~mm})$ to the organ level $(\geq 1 \mathrm{~cm})$. When the ultrasonic wavelength used to probe the tissues becomes comparable with the tissue's microstructure scale, the propagation and reflection of ultrasound waves cannot be fully interpreted employing classical models developed within the continuum assumption. In this study, we present a multiscale model for analyzing the mechanical response of a non-continuum double-layer system exposed to an ultrasound source. The model is developed within the framework of the Doublet Mechanics theory and can be applied to the non-invasive analysis of complex biological tissues.
\end{abstract}

Keywords: Nanomechanics; Doublet Mechanics; Ultrasound; Spectroscopy; Biopsy; Microscopic Elastography; Ultrasound Biomicroscopy

\section{INTRODUCTION}

In the last few years, there has been an increased interest to extend the limits of conventional clinical approaches to the level of microscopic resolution [1-5]. The goal here is to optimize the imaging of small tissue structures and, in general, to obtain information not available from the corresponding conventional macroscale applications.
The ability to quantitatively and non invasively differentiate living tissues based upon their biological and physical properties would enable major breakthroughs in the early detection and diagnosis of diseases and in monitoring therapeutic effects.

While surgical biopsy remains the 'gold standard' for the clinical screening of tissues and the assessment of pathologic conditions, there is a concerted effort to develop new imaging modalities that non-invasively visualize tissues providing information previously only available from biopsy [6-8]. Morphologic presentation of tissues together with the microbiologic, immunologic and molecular analysis are still critical for determining personalized medical treatments. However, the analysis of surgical biopsies suffers from being inherently operator dependent and, ultimately, the quality of diagnoses is entrusted with the pathologist's experience and knowledge [9-11]. Availability of quantitative imaging techniques capable of probing tissues at the microscale level may improve the accuracy of the diagnosis of biological samples and, most importantly, provide a new non-invasive means for the assessment of living tissues in situ.

Recent developments in the fields of optics, nuclear medicine, computed tomography, magnetic resonance and ultrasound have suggested the feasibility of obtaining tissue information at the micrometer-scale level with high accuracy, sensitivity and contrast-to-noise ratios. For example, optical in vivo biopsy is a growing area in optical computed tomography applications, which promises the assessment of tissue morphology and cell function as well as the detection of early-stage tissue abnormalities associated with diseases [7,12]. Similarly, new magnetic resonance microscopy techniques now permit imaging tissue's fine architecture in applications that range from assessing neural tissues [13] to imaging 
angiogenesis and gene expression in cancers [14,15]. Among the various imaging techniques, ultrasound methods have always offered distinctive characteristics, which make them particularly suitable for clinical screening of tissues. These include: cost-effectiveness, portability, non-invasiveness, and the ability to provide in vivo tissue clinical information in real-time, at high resolutions and relatively large depths.

Although the first acoustic microscope was proposed in the early 1930s [16], it was only in the late 1980s that the use of pulse-echo imaging systems operating at frequencies higher than the frequency range conventionally used in diagnostic imaging began to be experimented [17, 18]. Today, the ultrasonic visualization of tissues at microscopic resolution is usually referred to in the literature as ultrasound biomicroscopy or, more simply, high frequency ultrasound [4,6]. Important clinical applications of high frequency ultrasound techniques include: ophthalmology [19], dermatology [20], intravascular ultrasound [18], cartilage imaging [21], and obstetrics [22]. While the imaging performance of the ultrasound system is ultimately determined by the frequency of the ultrasonic transducer, its geometry and the tissue acoustic properties, the choice of the imaging system to be used in a given application is highly dependent on the nature of the application itself. For example, ophthalmic applications usually employ transducers with frequentcies in the 40 to $60 \mathrm{MHz}$ range [19]. These are used for imaging glaucoma, scleral and corneal diseases, and melanomas of the iris among the various clinical applications. The typical frequency range for investigating skin and assess skin tumor markers is from 20 to 40 $\mathrm{MHz}$ [20], with possible extension up to $100 \mathrm{MHz}$ [23]. For intravascular applications, ultrasound systems usually operate in the range of 20 to $30 \mathrm{MHz}$, allowing high resolution imaging of vessel walls and coronary arteries [24]. Finally, a new and promising application of high frequency ultrasound systems relates to the development of microscopic elasticity imaging and intravascular elastography imaging techniques, which use pre- and post-excitation high frequency ultrasound data to reconstruct maps of the microscopic mechanical properties of tissues [25-28].

A common denominator for all the aforementioned ultrasound applications is the use of high-frequency systems to probe the tissues so that high spatial resolutions can be achieved. However, biological tissues are non continuous, non homogeneous and exhibit a multiscale organization from the sub-cellular level $(\leq 1 \mathrm{~mm})$ to the organ level $(\geq 1 \mathrm{~cm})$. When the ultrasonic wavelength becomes comparable with the tissue's microstructure scale, the propagation and reflection of ultrasound waves cannot be fully interpreted employing classical models developed within the continuum assumption. In these regards, we have recently proposed the use of ultrasound-based Non Destructive Evaluation (NDE) techniques in conjunction with multiscale mathematical models as an integrated tool to automatically screen tissue biopsy specimens with high accuracy and resolution [29-32]. Biopsy samples are exposed to an ultrasound source and the tissue response and physical properties can be interpreted employing multiscale mathematical models. Normal and malignant tissues are expected to provide different responses that could be readily detected.

Several techniques have been proposed to model the mechanical behavior of materials at the nano/micro scale. This is the case for instance of the Lattice Dynamics (LD) and Molecular Dynamics [33], non-local Micromechanics theories of the differential (CGT) [34] and integral type (INT) [35-36]. In addition to these somehow classical approaches, the theory of Doublet Mechanics (DM) has been developed over the last twenty years as a multiscale field theory that allows to bridge the gap between Continuum Mechanics (CM) and discrete meso scale models without contradiction [37-39].

In this paper, we develop a mathematical model based on the DM approach to study the response of multilayered non continuum solids to ultrasounds. While the model is formulated for the analysis of biopsy samples, it may also be applied to the detection of malignant tumors developing in natural multi-layered systems such as the skin or the eye and might become an important tool for the further development of novel high frequency ultrasound elastography techniques.

\section{A LINEAR ELASTICITY BOUNDARY VALUE PROBLEM WITHIN DM}

The theory of Doublet Mechanics is a multi-scale theory which recapitulates Lattice Dynamics at the nanoscale limit and is fully compatible with the continuum mechanics framework at the macroscale limit. In the present paragraph, the governing equations for a linear elasticity problem are briefly recapitulated. A more detailed description can be found in [37-39].

Within the DM framework, a solid is considered as a spatial array of points (nodes) at finite distances. Any pair of adjacent nodes is termed a doublet comprising a reference node $X$ and a node $Y_{\alpha}$ located at a separation distance $\eta_{\alpha}^{o}$ and aligned along the doublet axis with unit vector $\tau_{\alpha}^{o}$ (Figure 1). A doublet is univocally identified by specifying its reference node $(X)$, orientation $\left(\tau_{\alpha}^{o}\right)$ and separation distance $\left(\eta_{\alpha}^{o}\right)$. The superscript $o$ means initial configuration. The node $X$ is surrounded by other $\mathrm{m}$ adjacent nodes, which form a number $m$ of doublets with corresponding $Y_{\alpha}$ nodes. 


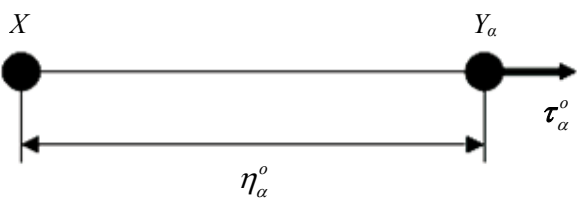

Figure 1. A doublet comprising a reference node $X$ and a node $Y$, at a separation distance $\eta$ aligned along the doublet axis indicated by the unit vector $\boldsymbol{\tau}$.

Such a set of nodes constitutes a bundle for the reference node $X$. If the nodes are arranged as to form one of the fourteen Bravais lattices, $m$ would coincide with the coordination number of the lattice and the internodal distances would coincide with the lattice constants.

Under externally applied loads, the nodes of a doublet are displaced giving rise to microdisplacements and microstrains. In general, if the node are separated along $\tau_{\alpha}^{o}$, the doublet undergoes an elongation $\varepsilon_{\alpha}$, if the nodes are rotated about $\tau_{\alpha}^{o}$ or separated normally to $\tau_{\alpha}^{o}$, the doublet undergoes a torsion $\mu_{\alpha}$ or a shear $\gamma_{\alpha}$, respectively. In the sequel, it is assumed that each doublet can only undergo elongations (central interactions) which would be associated with the build up of a microstress $p_{\alpha}$ along $\tau_{\alpha}^{o}$.

An orthogonal Cartesian frame of reference is introduced with unit vectors $\boldsymbol{e}_{i}(i=1,2,3)$, and each node $X$ is associated to a position vector $\boldsymbol{x}=x_{i} \boldsymbol{e}_{i}$, where the classical convention of the repeated Roman indices is used. A displacement vector $\boldsymbol{u}_{\alpha}(\boldsymbol{x} ; t)$ can be introduced and for each doublet; the increment displacement vector $\Delta \boldsymbol{u}_{\alpha}(\boldsymbol{x} ; t)$ can be defined as the difference between the displacement of the node $\gamma_{\alpha}$ and that of the node $X$ at time $t$

$$
\Delta \boldsymbol{u}_{a}=\boldsymbol{u}\left(Y_{\alpha}\right)-\boldsymbol{u}(X)=\boldsymbol{u}\left(\boldsymbol{x}+\eta_{\alpha}^{o} \tau_{\alpha}^{o}, t\right)-\boldsymbol{u}(\boldsymbol{x}, t)
$$

As in linear elasticity, it is assumed that the relative displacement $\left|\Delta \boldsymbol{u}_{a}(\boldsymbol{x} ; t)\right|$ is small compared to the doublet separation distance $\eta_{\alpha}^{o}\left(\left|\Delta \boldsymbol{u}_{a}(\boldsymbol{x} ; t)\right| \ll \eta_{\alpha}^{o}\right)$ so that the initial and final configuration of the system can be as- sumed to coincide. Expanding $\Delta \boldsymbol{u}_{a}$ in a convergent Taylor series in a neighborhood of the reference point $X$, it follows, in scalar form

$$
\Delta u_{a_{i}}=\sum_{\chi=1}^{M} \frac{\left(\eta_{\alpha}^{o}\right)^{\chi}}{\chi !} \tau_{\alpha k_{1}}^{o} \cdots \tau_{\alpha k_{\chi}}^{o} \frac{\partial^{\chi} u_{i}}{\partial x_{k_{1}} \cdots \partial x_{k_{\chi}}}
$$

where each of the subscript $k_{1}, \cdots, k_{\chi}$ runs through 1 to 3 . The order $M$ at which the series is truncated defines the degree of approximation employed by the DM theory. For $M=1$, the continuum theory of elasticity is recovered.

The small elongation of the doublet $\varepsilon_{\alpha}$ can be defined as

$$
\varepsilon_{\alpha}=\frac{\Delta \boldsymbol{u}_{\alpha} \cdot \tau_{\alpha}^{o}}{\eta_{\alpha}}
$$

and, using (2), Equation (3) can be rephrased as

$$
\varepsilon_{\alpha}=\tau_{\alpha i}^{o} \sum_{\chi=1}^{M} \frac{\left(\eta_{\alpha}^{o}\right)^{\chi-1}}{\chi !} \tau_{\alpha k_{1}}^{o} \cdots \tau_{\alpha k_{\chi}}^{o} \frac{\partial^{\chi} u_{i}}{\partial x_{k_{1}} \cdots \partial x_{k_{\chi}}}
$$

which can be interpreted as the compatibility equation within the linear DM theory. The relationship between the doublet microstress $p_{a}$ and microstrain $\varepsilon_{\alpha}$ is given as

$$
p_{\alpha}=A_{\alpha \beta} \varepsilon_{\beta}
$$

in the case of linear and homogeneous internodal central interactions. Equation (5) can be interpreted as the constitutive equation in the linear and homogeneous DM theory, and $A_{\alpha \beta}$ is the matrix of the homogeneous micro modulii of the doublet. Finally, static equilibrium is imposed as

$$
\begin{aligned}
& \sum_{\alpha=1}^{m / 2} \sum_{\chi=1}^{M}(-1)^{\chi-1} \frac{\left(\eta_{\alpha}^{o}\right)^{\chi-1}}{\chi !} \tau_{\alpha k_{1}}^{o} \cdots \tau_{\alpha k_{\chi}}^{o} \frac{\partial^{\chi}\left(p_{\alpha_{i}}\right)}{\partial x_{k_{1}} \ldots \partial x_{k_{\chi}}}+F_{i}=0 \\
& i=1,2,3
\end{aligned}
$$

where $F_{i}$ are the scalar components of the volume force $\boldsymbol{F}$. The boundary conditions expressed in terms of stresses take the form

$$
\begin{aligned}
& n_{k_{r}} \sum_{\alpha=1}^{m / 2} \tau_{\alpha k_{r}}^{o} \sum_{\chi=r}^{M}(-1)^{\chi-1} \frac{\left(\eta_{\alpha}^{o}\right)^{\chi-1}}{\chi !} \\
& \quad \times \tau_{\alpha k_{r+1}}^{o} \cdots \tau_{\alpha k_{\chi}}^{o} \frac{\partial^{\chi-r}\left(p_{\alpha_{i}}\right)}{\partial x_{k_{r+1}} \ldots \partial x_{k_{\chi}}}=T_{i} \delta_{r 1} i=1,2,3 ;
\end{aligned}
$$

where $n_{k r}$ are the scalar components of the unit vector $\boldsymbol{n}$ normal to the body surface, and the subscript $r=1, \cdots$, $M-1$ for $M \geq 2$ and $r=1$ for $M=1 . T_{i}$ are the scalar components of the vector force $\boldsymbol{T}$. The term $\boldsymbol{d}_{r 1}$ is the Kronecker delta function. The Equations (4), (5) and (6) together with (7) give a boundary value problem within DM for a linear elastic body. Notice that Equations (6) and (7) are general to the extent that they are written as a function of the doublet unit vectors $\tau_{\alpha i}$.

The relationship between the micro- and the macrostresses has been derived in [37] as

$$
\sigma_{k_{1} i}^{(M)}=\sum_{\alpha=1}^{m / 2} \tau_{\alpha k_{1}}^{o} \sum_{\chi=1}^{M}(-1)^{\chi-1} \frac{\left(\eta_{\alpha}\right)^{\chi-1}}{\chi !} \tau_{\alpha k_{2}}^{o} \cdots \tau_{\alpha k_{\chi}}^{o} \frac{\partial^{\chi-1}\left(p_{\alpha i}\right)}{\partial x_{k_{2}} \cdots \partial x_{k_{\chi}}}
$$

at a generic level of approximation $M$. 


\section{ULTRASOUND WAVE PROPAGATION IN BIOLOGICAL TISSUES}

A thin slice of biological tissue is considered (histologycal sample) embedded between two glass slides. An auxiliary continuous layer is placed upon the biological slice, as in Figure 2 to simulate a multilayered complex tissue system. An ultrasound transducer is used to probe this sandwich-like structure, generating mechanical waves of both shear and longitudinal type. The initial train of waves (0) travels unperturbed in the $\theta_{0}$ direction (Figure 3 , see also Appendix A). Upon the interaction of (0) with the first glass layer four more waves are generated (assuming specular reflection): two are forward scattered into the system (waves (3) and (4)) and two are back scattered into the glass substrate (waves (1) and (2)). Similarly, other waves are generated at the interface between the auxiliary layer and the tissue layer at the interface between the tissue and the second glass layer. Under the approximation of neglecting multiple reflections higher than the second order, the total number of waves travelling within the system is thirteen for the present configuration (Figure 3). Theoretically, a comparison between the interacting waves at each interface would allow to deduce the reflection coefficients, given by the ratio between the amplitudes of the incident and reflecting waves.

In the sequel, the DM theory is used to model the heterogeneous biological tissue and the auxiliary continuous layer, for which a scaleless approximation $(M=1$ : Continuum Mechanics) would be sufficient. In particular, the reflection coefficients are derived. Details of these derivations are given in Appendix A. According to the theory derived in this study, the reflection coefficient at the first interface, $R_{1}$, is a complex function of the internodal distance $\eta$, the doublets configuration embodied by t's, the elastic microconstants $A_{\alpha \beta}$. Thus, estimation of the reflection coefficients can provide important information regarding the tissue microstructure and mechanical properties. Since different tissues are expected to exhibit different responses, spectral analysis of the reflection coefficients may allow differentiation between different tissue types as well as monitoring changes occurring in the microstructural properties of a tissue due to pathology. In the next section, we analyze two distinctive cases where the application of the developed theory is used for the ultrasonic characterization of a biological tissue: a mono-layer model with a single tissue, and a double-layer model comprising a biological slice and an auxiliary continuum layer.

\subsection{The Mono-Layer Model}

In the limit that the thickness of the auxiliary layer is going to zero $\left(h_{1} \rightarrow 0\right)$ a mono-layer, discrete model is

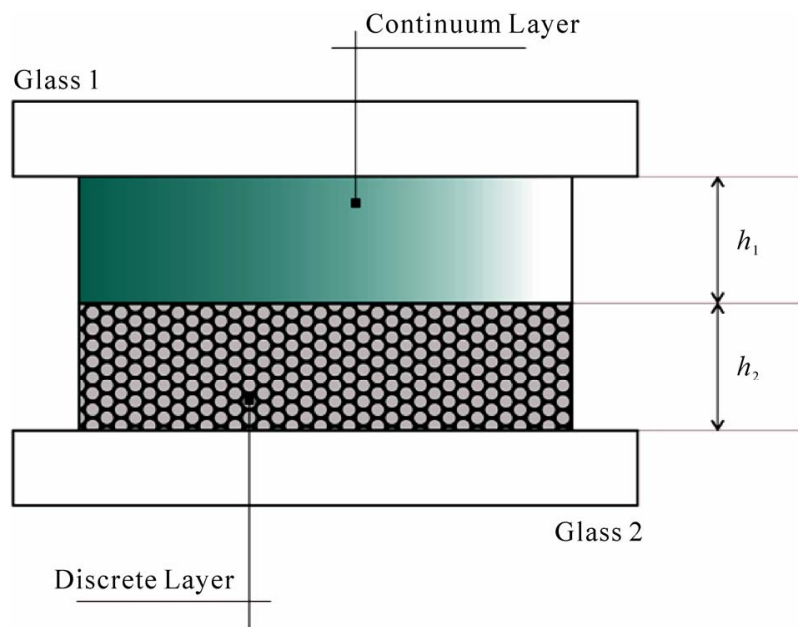

Figure 2. The sandwhich like structure comprising an intermediate continuum layer, a biological discrete tissue substrate and two external glass dishes. The system is feasible to be tested through ultrasounds.

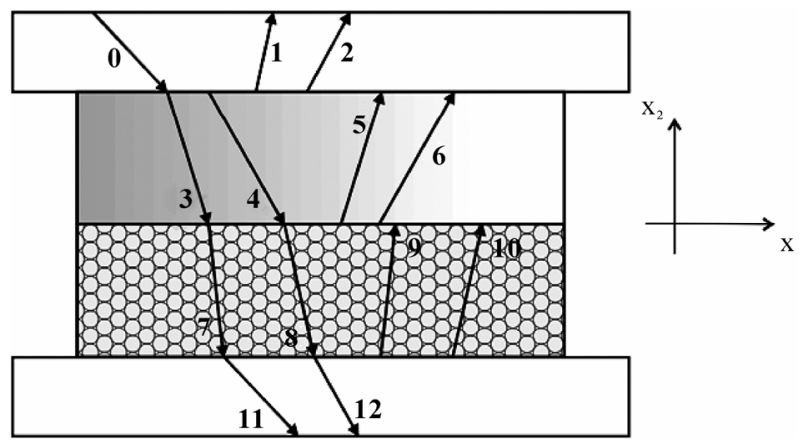

Figure 3. Elastic waves propagating in the system.

obtained as in [29-32]. Figure 4 shows a plot of the reflection coefficient $\mathrm{R}_{1}$ as a function of the frequency $f$ of the ultrasound transducer. For comparison, a plot of the reflection coefficient as obtained using the CM theory as opposed to the DM theory is also shown. These plots are obtained by imposing $\eta=0, \lambda=1.805 \mathrm{GPa}, \mu=0.04875$ $\mathrm{GPa}$, and the DM solution, with $\eta=5 \mu \mathrm{m}$, and $A_{11}=2$ $\mathrm{GPa}, A_{44}=0.195 \mathrm{GPa}$ as derived using $\lambda$ and $\mu$ in equation (15). These parameters are set based on previous studies retrievable in the literature [29-32,40]. These results show that at low frequencies, smaller than about $10 \mathrm{MHz}$, the CM and DM solutions tend to overlap without any significant difference; whereas at higher frequencies, significant discrepancies emerge. This may be explained by observing that, at low frequencies, the probing waves have a finite length that is greater than the characteristic length scale of the system $\eta$ (tens of microns against $\eta=5 \mu \mathrm{m})$. Consequently the micro structure of the layer is averaged out within the ultrasonic wavelength, which ultimately limits the spatial resolution, and the predictions of CM and DM coincide. 


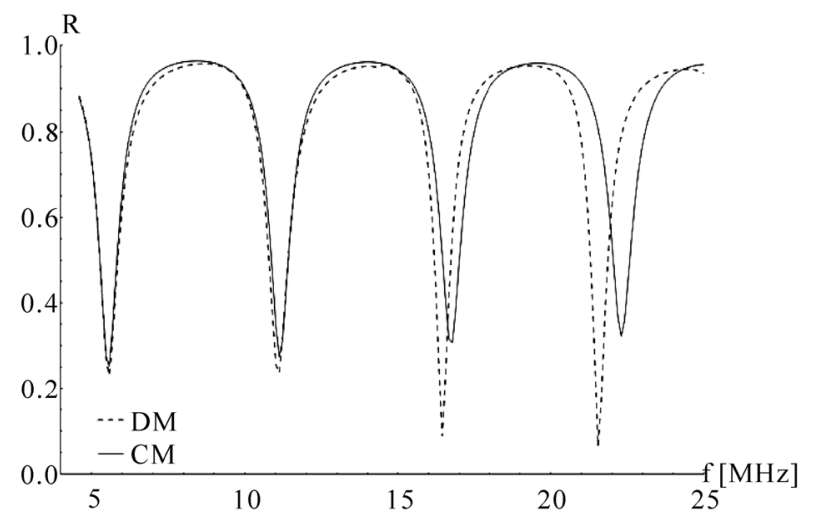

Figure 4. The reflection coefficient $\mathrm{R}_{1}$ shown against the frequency $f$ and comparison between the $\mathrm{CM}$ solution.

Notice that $\eta$ has the same order of magnitude of a cell size. Conversely, as $f$ increases, and the ultrasonic wavelengths become comparable to the scale length of the small components of the material (cells), the difference between the discrete and continuum approach becomes more and more evident.

The relation between the angular frequency $\omega$, the wave number $k_{i}$ and the phase velocity $c_{i}$ in the framework of DM is embodied by the quite complex equations (18) (Appendix A). Interestingly, these relations reduce to the non scale, classical dispersion ones (where simple relations $k_{i}=\omega / c_{i}, c_{i}=(E / \rho)^{1 / 2}$ hold true) via two different assumptions: 1) the constituent granules of the biological substrate are material points whose sizes are infinitesimal $\eta=0$ and all the waves may have arbitrary but finite length, 2) the constituent particles of the biological substrate may have an arbitrary but finite size $(\eta>0)$ and all the waves have an infinite length $(k \rightarrow 0)$. The diagrams of Figure 4 show the occurrence of a number of minima and maxima. These can be explained considering phenomena of interference occurring at each interface between the reflecting and transmitting waves.

\subsection{The Double Layer Model}

Herein the results stemming out from the double layer model are presented, where both $h_{1}$ and $h_{2}$ are different from zero. For the analysis, it is assumed that the auxiliary layer is made up of a polymeric material commonly used in biomedical applications (Table 1). The biological tissue has the properties listed in Table 2. These parameters are set based on previous studies retrievable in literature $[29-32,40]$. The CM and DM frameworks are used in describing the auxiliary and biological substrate, respectively. In Figures from 5 to 9, the reflection spectra of the system under study are shown for different auxiliary layer materials, with varying Young's modulus $E$ and for wave frequencies ranging from 4 to $12 \mathrm{MHz}$. It can be concluded that as $E$ increases, the number of
Table 1. The CM properties of the intermediate layer.

\begin{tabular}{ccccc}
\hline Material & $\begin{array}{c}\rho \\
{\left[10^{3} \mathrm{~kg} / \mathrm{m}^{3}\right]}\end{array}$ & $\begin{array}{c}\mathrm{E} \\
{[\mathrm{GPa}]}\end{array}$ & $v$ & $\begin{array}{c}\mathrm{h} \\
{[\mathrm{mm}]}\end{array}$ \\
\hline PDMS & 0.97 & $4.8 \times 10^{-4}$ & 0.49 & 0.133 \\
Polyurethane & 1.10 & $5.3 \times 10^{-3}$ & 0.48 & 0.133 \\
Polyethylene & 0.955 & 0.70 & 0.41 & 0.133 \\
Polypropylene & 0.91 & 1.30 & 0.42 & 0.133 \\
Polycarbonate & 1.23 & 2.40 & 0.41 & 0.133 \\
\hline
\end{tabular}

Table 2. The CM properties of the glass substrates, and the DM parameters of the biological tissue.

\begin{tabular}{cccccc}
\hline Material & $\begin{array}{c}\rho \\
{\left[10^{3} \mathrm{~kg} / \mathrm{m}^{3}\right]}\end{array}$ & $\begin{array}{c}\mathrm{E} \\
{[\mathrm{GPa}]}\end{array}$ & $v$ & $\begin{array}{c}\mathrm{h} \\
{[\mathrm{mm}]}\end{array}$ & \\
\cline { 1 - 5 } CROWN glass & 2.50 & 71.8 & 0.23 & 0.133 & \\
\cline { 1 - 5 } Material & $\begin{array}{c}\rho \\
{\left[10^{3} \mathrm{~kg} / \mathrm{m}^{3}\right]}\end{array}$ & $\begin{array}{c}\mathrm{A}_{11} \\
{[\mathrm{GPa}]}\end{array}$ & $\begin{array}{c}\mathrm{A}_{44} \\
{[\mathrm{GPa}]}\end{array}$ & $\begin{array}{c}\mathrm{h} \\
{[\mathrm{mm}]}\end{array}$ & $\begin{array}{c}\eta \\
{[\mathrm{mm}]}\end{array}$ \\
\hline Bio tissue & 0.95 & 2.20 & 0.36 & 0.133 & 5.6 \\
\hline
\end{tabular}

minima decreases within the frequency range considered, as it does the difference in frequency between two successive minima. Most importantly, at high values of $E$, the minimum values of the reflection coefficient do not vary significantly. Since increased tissue stiffness is often associated with changes in tissue pathology, spectral analysis of reflection coefficients as interpreted within the DM theory may contain important markers for the assessment of a tissue state and its changes due to the onset of diseases. These simulations also show that materials with a high compliances, such as PDMS (Figure 5), generate a very complex response, with several minima. Such complexity would spoil the reflection signal, inducing more noise and making more difficult the accurate interpretation of the spectra. Stiffer intermediate layers would be more convenient. Additional simulation (Figure 10) shows the effects of varying the Young's modulus $(\Delta E= \pm 10 \%)$ about the mean value for the Polyethylene case. As $E$ increases, the spectrum undergoes a rigid translation towards higher frequencies (see Table 3 for the complete list of values).

The effect of thickness $h_{1}$ of the continuum, intermediate layer has been also investigated. Starting from a thickness of $134 \mu \mathrm{m}$, a $10 \%$ variation has been imposed (Polyethylene, $\Delta h= \pm 10 \%$ ) and the reflection spectra evaluated. Results are shown in Figure $\mathbf{1 1}$ and Table 4. It is observed that as $h_{1}$ increases, the spectra rigidly move towards lower frequencies, with no change in the number of minima. The thickness $h_{1}$ also affects the damping of the system, the thicker is the intermediate layer and the stronger is the attenuation for the spectra. 


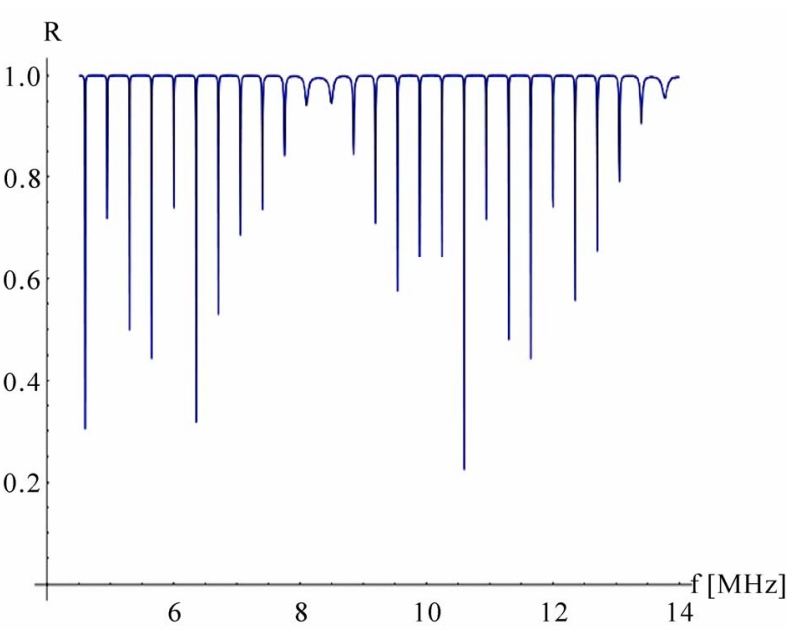

Figure 5. The reflection spectrum of the system assuming that the intermediate layer is PDMS.

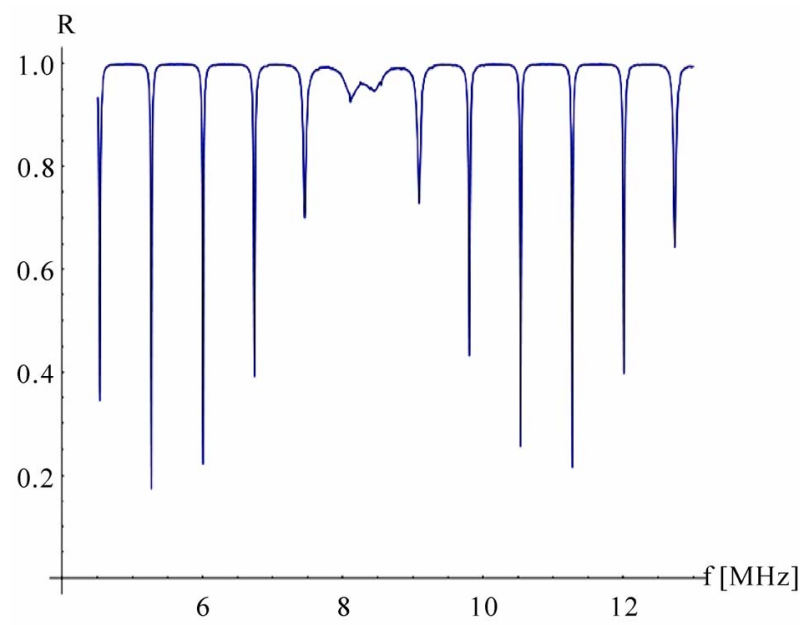

Figure 6. The reflection spectrum of the system assuming that the intermediate layer is PU.

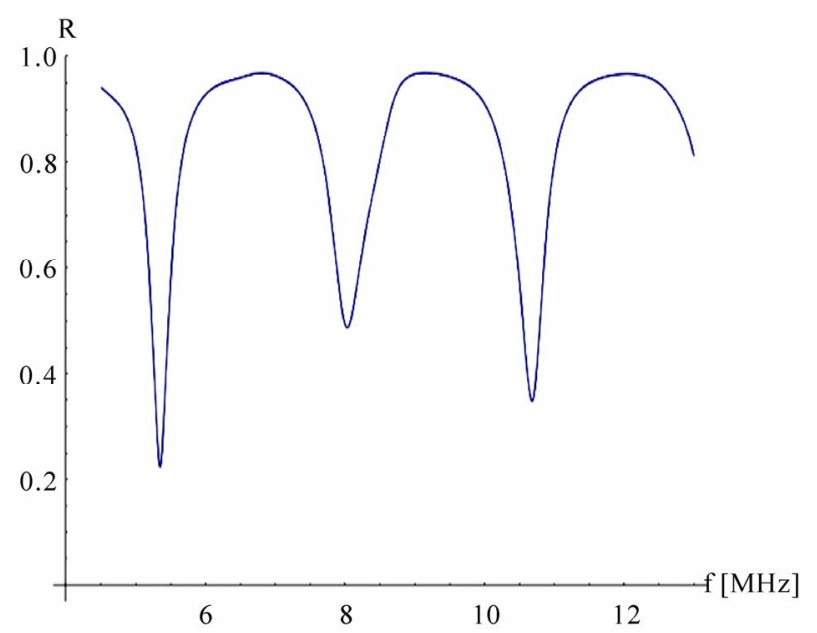

Figure 7. The reflection spectrum of the system assuming that the intermediate layer is PE.

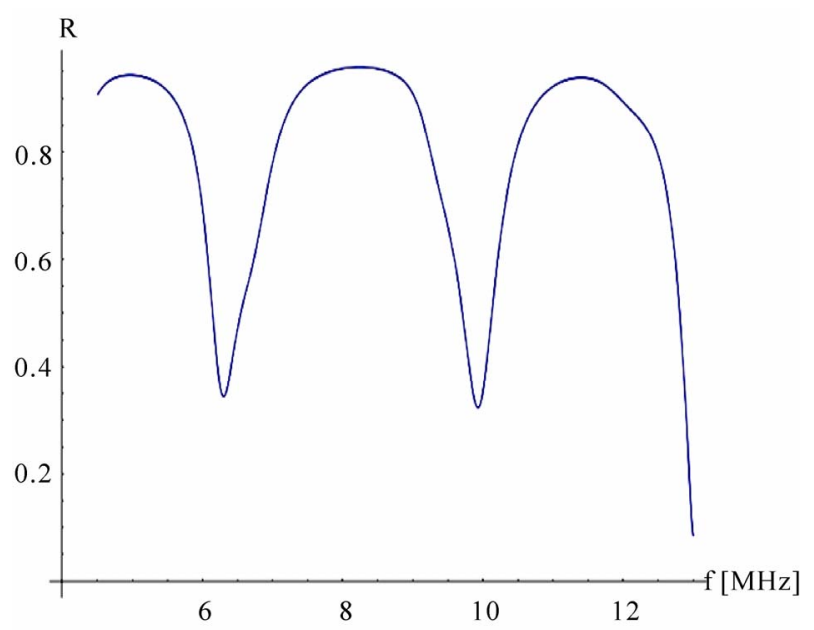

Figure 8. The reflection spectrum of the system assuming that the intermediate layer is PP.

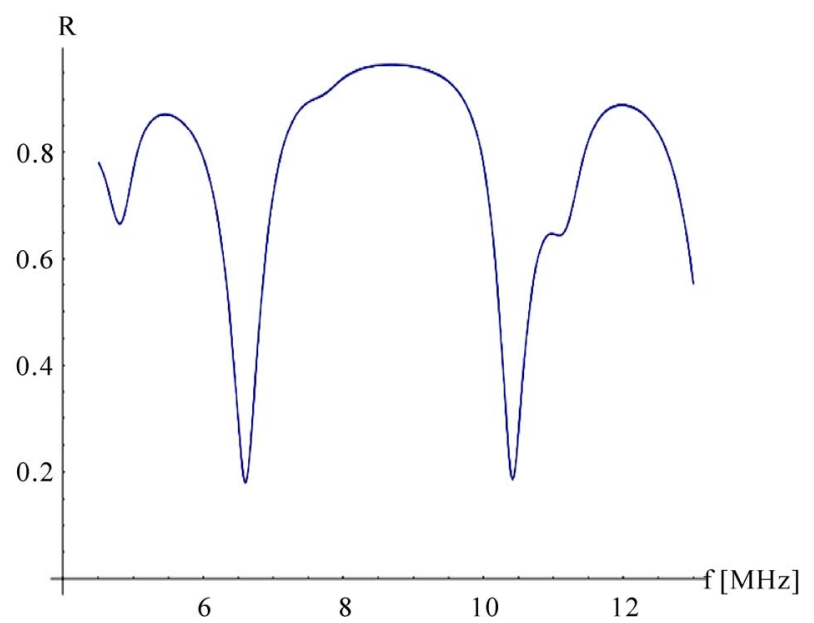

Figure 9. The reflection spectrum of the system assuming that the intermediate layer is PC.

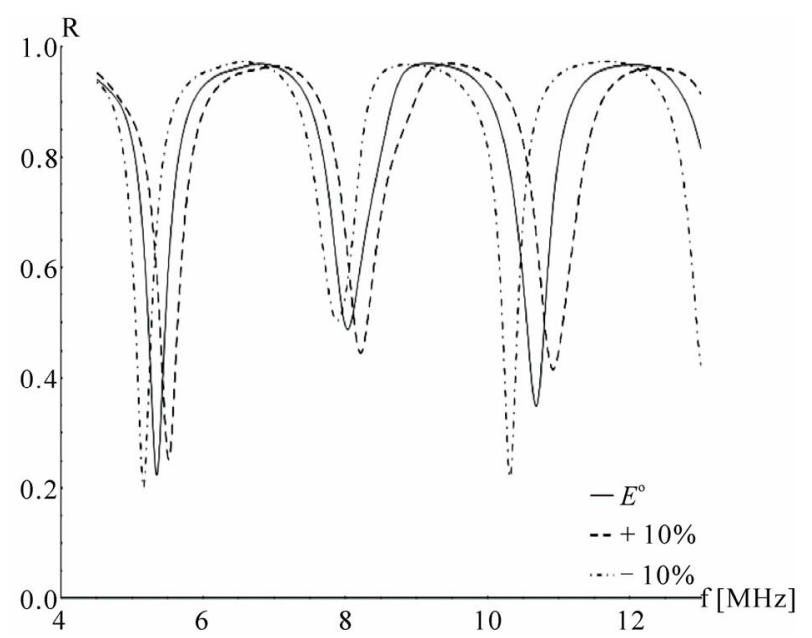

Figure 10. The effect of the variation of the Young's modulus E upon the overall response of the system. 
Table 3. Characterist minima points for different plastic substrates.

\begin{tabular}{|c|c|c|c|c|c|c|c|c|c|}
\hline \multicolumn{2}{|c|}{ PDMS } & \multicolumn{2}{|c|}{ PU } & \multicolumn{2}{|c|}{ PE } & \multicolumn{2}{|c|}{ PP } & \multicolumn{2}{|c|}{ PC } \\
\hline$f[\mathrm{MHz}]$ & $\mathrm{R}$ & $f[\mathrm{MHz}]$ & $\mathrm{R}$ & $f[\mathrm{MHz}]$ & $\mathrm{R}$ & $f[\mathrm{MHz}]$ & $\mathrm{R}$ & $f[\mathrm{MHz}]$ & $\mathrm{R}$ \\
\hline 4.595 & 0.333 & 4.534 & 0.334 & 5.348 & 0.224 & 6.301 & 0.342 & 6.602 & 10.418 \\
\hline 4.946 & 0.282 & 5.269 & 0.186 & 8.031 & 0.487 & 9.930 & 0.322 & 0.181 & 0.187 \\
\hline 5.298 & 0.311 & 6.005 & 0.192 & 10.681 & 0.347 & & & & \\
\hline 5.650 & 0.391 & 6.739 & 0.363 & & & & & & \\
\hline 6.002 & 0.411 & 7.457 & 0.684 & & & & & & \\
\hline 6.354 & 0.409 & 8.109 & 0.921 & & & & & & \\
\hline 6.705 & 0.446 & 8.456 & 0.949 & & & & & & \\
\hline 7.055 & 0.510 & 9.091 & 0.738 & & & & & & \\
\hline 7.405 & 0.677 & 9.806 & 0.431 & & & & & & \\
\hline 7.753 & 0.835 & 10.538 & 0.235 & & & & & & \\
\hline 8.101 & 0.940 & 11.274 & 0.211 & & & & & & \\
\hline 8.501 & 0.946 & 12.009 & 0.347 & & & & & & \\
\hline 8.847 & 0.850 & & & & & & & & \\
\hline 9.193 & 0.710 & & & & & & & & \\
\hline 9.543 & 0.578 & & & & & & & & \\
\hline 9.894 & 0.421 & & & & & & & & \\
\hline 10.246 & 0.327 & & & & & & & & \\
\hline 10.597 & 0.252 & & & & & & & & \\
\hline 10.949 & 0.200 & & & & & & & & \\
\hline 11.300 & 0.240 & & & & & & & & \\
\hline 11.652 & 0.315 & & & & & & & & \\
\hline 12.003 & 0.365 & & & & & & & & \\
\hline
\end{tabular}

Table 4. Characterist minima points for different plastic substrates.

\begin{tabular}{|c|c|c|c|c|c|c|c|c|c|}
\hline \multirow{2}{*}{$\min$} & \multicolumn{3}{|c|}{ Initial E } & \multicolumn{3}{|c|}{$+10 \%$} & \multicolumn{3}{|c|}{$-10 \%$} \\
\hline & 1 & 2 & 3 & 1 & 2 & 3 & 1 & 2 & 3 \\
\hline$f[\mathrm{MHz}]$ & 5.348 & 8.031 & 10.681 & 5.514 & 8.214 & 10.919 & 5.160 & 7.883 & 10.315 \\
\hline $\mathrm{R}$ & 0.224 & 0.487 & 0.347 & 0.252 & 0.445 & 0.415 & 0.203 & 0.503 & 0.221 \\
\hline
\end{tabular}

Notice that the thickness $h_{1}$ and the stiffness $E$ have opposite effects.

\section{DISCUSSIONS}

In this study, we have presented a multiscale model for analyzing the mechanical response of a non-continuum system exposed to an ultrasound source. The proposed model may be interpreted as a quantitative, non-invasive ultrasound spectroscopy method, which is based on the analysis of the reflection coefficients as a function of the ultrasonic wavelength (or conversely the frequency) for estimating changes in the tissue microstructure and mechanical properties. The model was developed within the framework of the Doublet Mechanics theory, which led to the determination of the reflection coefficients as a function of the internodal distance, the doublets configuration and the elastic microconstants. In its present form, the model does not take into consideration scattering effects at the various interfaces or within the tissue layer. Coupling this effect with the DM theory would 


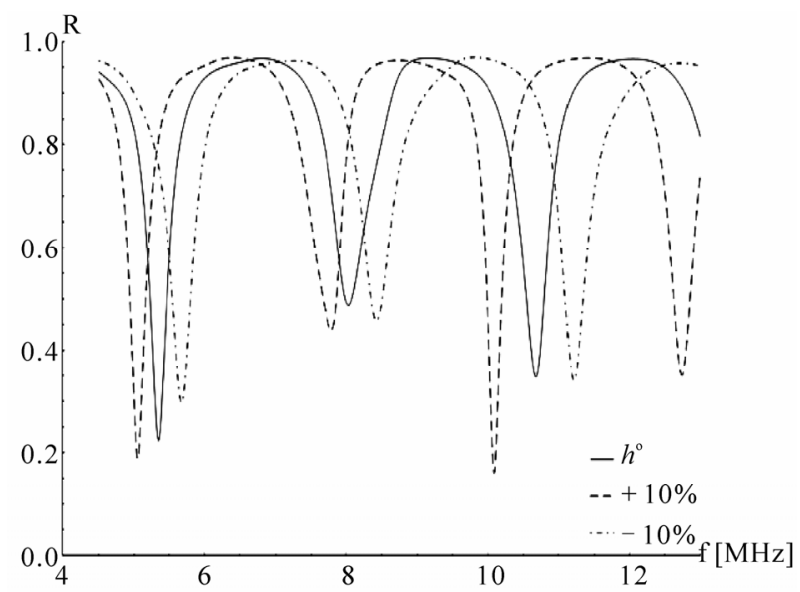

Figure 11. The effect of the variation of the intermediate layer thickness $h_{1}$ upon the overall response of the system.

require large mathematical efforts, which are considered outside the scope of the presented study and left for future investigations. In Appendix B, we provide a detailed analysis of the conditions under which this approximation is valid.

As a test platform, the DM model developed in this study was applied to the ultrasonic characterization of two tissue models: a mono-layer solid simulating a tissue specimen for histological analysis and a double-layer system simulating a more complex tissue. For the mono-layer case, the results obtained using the DM theory were statistically compared with the results obtained from the same tissue model using the classical CM theory. The results reported in this study demonstrate that at frequencies as high as $10 \mathrm{MHz}$ and above, the $\mathrm{CM}$ results significantly deviate from the DM results. This would suggest that the use of CM-based approaches to characterize the ultrasonic behavior of tissues exposed to high frequency $(>10 \mathrm{MHz})$ insonications may lead to incorrect or incomplete interpretation of the ultrasonic parameters of interest with respect to the tissue microstructural properties. In such experimental conditions, a more complicated model should be considered. The model described in this paper offers an attractive alternative, which is based on the spectral analysis of the receiving signals to differentiate between tissue types. In addition, the analysis of the single layer tissue model carried out in this paper may prove as a useful example on how to practically apply the proposed DM model to ultrasound-based histological applications.

The present work with the development of a double layer mathematical model constitutes the first step towards a more realistic representation of natural multilayered systems as the human skin and the growth and spread of malignancies such as melanoma. Human skin is a unique organ that permits life by regulating heat and water loss from the body while preventing the ingress of noxious chemicals or microorganisms [41]. Skin membranes may be examined at various levels of complexity. While the membrane is regarded sometimes as a simple physical barrier, more complexity may be introduced by considering skin as various layers in series, namely 1) the innermost subcutaneous fat layer (hypodermis); 2) the overlying dermis; 3) the viable epidermis; 4) the outermost layer of the tissue (the stratum corneum). All the cited layers posses, at different extents, a certain degree of heterogeneity due to sebaceous glands, hair follicles, fat lobules, blood vessels, nucleii and desmosomal junctions [41]. With such a scenario, a simple singlelayer model would not be sufficient for interpreting the system response. In addition, skin cancer is associated with localized changes in the tissue microstructure and morphological modification at the interfaces between different skin layers making single-layer models potentially inaccurate in predicting the onset and spread of tumor masses.

As a demonstration of the practical applicability of some of the concepts exposed in this paper, we have previously developed an ex vivo apparatus that uses ultrasound technology and the Doublet Mechanics theory to obtain information about tissue pathological states $[31,40]$. For the purpose of illustration, a sketch of such apparatus and set-up is provided in Figure 12. The ultrasonic wand delivers an acoustical wave of known frequency into the tissue, and then detects the reflected wave fractions with separate transducers. As described in this study, a characteristic reflection spectrum can be plotted depicting the reflection coefficient versus the

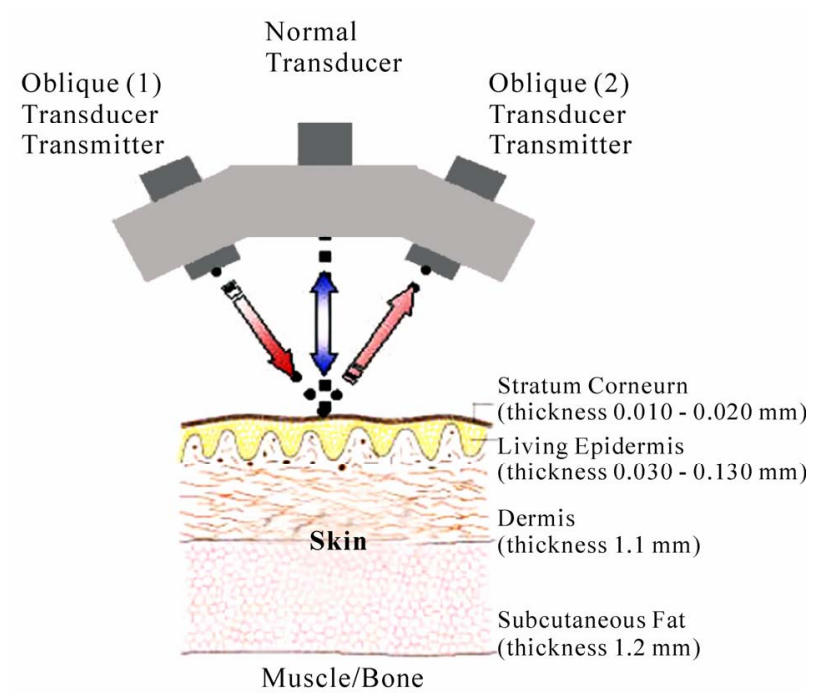

Figure 12. A sketch of an ex vivo apparatus based on ultrasound technology and the Doublet Mechanics theory for the determination of the malignancy potential of a cutaneous or mucousal growth such as melanoma. 
excitation frequency. The spectrum can be related to tissue physical properties such as density, microelastic constants, attenuation, and internodal distance via the DM theory. Through comparative analysis of reflection spectra from normal and diseased tissue, it may be possible to determine the malignancy potential of a cutaneous or mucousal growth such as melanoma. This would allow physicians to screen patients on an annual or semi-annual basis for the presence of dysplastic nevi (pre-cancerous) or early stage cancerous skin lesions.

In addition to dermatology applications, the proposed model may have significant potentials in several other clinical applications that employ high frequency ultrasound systems for the assessment of tissue pathological and physiological states. It may be particularly useful in ophthalmology, where the anatomy of the eye resembles a multilayered system and where the use of high frequency ultrasound techniques has already shown significant potentials for the diagnosis and treatment of eye diseases [19]. Other possible applications may include cartilage assessment, intravascular applications, and high frequency elastography techniques, which ultimately long to the quantitative estimation of the tissue mechanical behavior at the microscopic scale.

\section{CONCLUSIONS}

The Doublet Mechanics theory has been employed to model the propagation of elastic waves within a biological tissue. The present case is characterized by heterogeneous and not continuous materials that, consequently, may be conveniently analyzed within the multiscale DM framework.

It has been observed that an operating frequency of the ultrasound generator increases, the difference between the DM solution and the classical elasticity solution becomes larger, as a consequence of the intimate interaction among waves and microscopic components of the biological tissue. It has been discussed that a DM multilayer model could be effective in describing the skin multi-layer structure. The design requirements for a characterization-mode ultrasound skin cancer detection system should provide primary care physicians with a rapid, noninvasive, screening tool for malignant melanoma that would assign a quantitative malignancy potential for specific cutaneous lesions.

\section{REFERENCES}

[1] Rajadhyaksha, M., Grossman, M., Esterowitz, D. and Webb, R.H. (1995) In vivo confocal scanning laser microscopy of human skin-melanin provides strong contrast. Journal of Investigative Dermatology, 104, 946-952. doi:10.1111/1523-1747.ep12606215

[2] Cross, S.E., Jin, Y.S., Rao, J. and Gimzewski, J.K. (2007)
Nanomechanical analysis of cells from cancer patients. Nature Nanotechnology, 2, 780-783.

doi:10.1038/nnano.2007.388

[3] Ellegala, D.B., Poi, H.L., Carpenter, J.E., Klibanov, A.L., Kaul, S., Shaffrey, M.E., Sklenar, J. and Lindner, J.R. (2003) Imaging tumor angiogenesis with contrast ultrasound and microbubbles targeted to $\alpha_{\mathrm{v}} \beta_{3}$. Circulation, 108, 336-341. doi:10.1161/01.CIR.0000080326.15367.0C

[4] Foster, F.S., Pavlin, C.J., Lockwood, G.R., Ryan, L.K., Harasiewicz, K.A., Berube, L. and Rauth, A.M. (1993) Principles and applications of ultrasound backscatter microscopy. IEEE Transactions on Ultrasonics Ferroelectrics and Frequency Control, 40, 608-617. doi:10.1109/58.238115

[5] Othman, S.F., Xu, H.H., Royston, T.J. and Magin, R.L. (2005) Microscopic magnetic resonance elastography ( $\mu \mathrm{MRE})$. Magnetic Resonance in Medicine, 54, 605-615. doi: $10.1002 / \mathrm{mrm} .20584$

[6] Foster, F.S., Pavlin, C.J., Harasiewicz, K.A., Christopher, D.A. and Turnbull, D.H. (2000) Advances in ultrasound biomicroscopy. Ultrasound in Medicine and Biology, 26, 1-27. doi:10.1016/S0301-5629(99)00096-4

[7] Tearney, G.J., Brezinski, M.E., Bouma, B.E., Boppart, S.A., Pitris, C., Southern, J.F. and Fujimoto, J.G. (1997) In vivo endoscopic optical biopsy with optical coherence tomography. Science, 276, 2037-2039. doi:10.1126/science.276.5321.2037

[8] Sonn, G.A., Jones, S.N.E., Tarin, T.V., Du, C.B., Mach, K.E., Jensen, K.C. and Liao, J.C. (2009) Optical biopsy of human bladder neoplasia with in vivo confocal laser endomicroscopy. Journal of Urology, 182, 1299-1305. doi:10.1016/j.juro.2009.06.039

[9] Paradiso, A. et al. (2004) Interobserver reproducibility of immunohistochemical HER-2/neu evaluation in human breast cancer: The real-world experience. International Journal of Biological Markers, 19, 147-154.

[10] Verkooijen, H. et al. (2003) Interobserver variability between general and expert pathologists during the histopathological assessment of large-core needle and open biopsies of non-palpable breast lesions. European Journal of Cancer, 39, 2187-2191. doi:10.1016/S0959-8049(03)00540-9

[11] Schlemper, R. et al. (2000) Differences in diagnostic criteria for esophageal squamous cell carcinoma between Japanese and Western pathologists. Cancer, 88, 996-1006. doi:10.1002/(SICI)1097-0142(20000301)88:5<996::AID -CNCR8>3.0.CO;2-Q

[12] Fercher, A.F., Drexler, W., Hitzenberger, C.K. and Lasser, T. (2003) Optical coherence tomography-principles and applications. Reports on Progress in Physics, 66, 239303. doi:10.1088/0034-4885/66/2/204

[13] Benveniste, H., Hedlund, L.W. and Johnson, G.A. (1992) Mechanism of detection of acute crebral-ischemia in rats by diffusion-weighted magnetic-resonance microscopy. Stroke, 23, 746-754.

[14] McDonald, D.M. and Choyke, P.L. (2003) Imaging of angiogenesis: From microscope to clinic. Nature Medicine, 9, 713-725. doi:10.1038/nm0603-713

[15] Louie, A.Y., Huber, M.M., Ahrens, E.T., Rothbacher, U., Moats, R., Jacobs, R.E., Fraser, S.E. and Meade, T.J. (2000) In vivo visualization of gene expression using magnetic resonance imaging. Nature Biotechnology, 18, 
321-325. doi:10.1038/73780

[16] Sokolov, S.J. (1935) Ultrasonic oscillations and their applications. Technical Physics of the USSR, 2, 522-534.

[17] Sherar, M.D., Noss, M.B. and Foster, F.S. (1987) Ultrasound backscatter microscopy images the internal structure of living tumour spheroids. Nature, 330, 493-495. doi:10.1038/330493a0

[18] Gussenhoven, E.J., Essed, C.E., Lancee, C.T., Mastik, F., Frietman, P., Vanegmond, F.C., Reiber, J., Bosch, H., Vanurk, H., Roelandt, J. and Bom, N. (1989) Arterial wall characteristics determined by intravascular ultrasound imaging: An in vitro study. Journal of the American College of Cardiology, 14, 947-952. doi:10.1016/0735-1097(89)90471-3

[19] Pavlin, C.J., Harasiewicz, K., Sherar, M.D. and Foster, E.S. (1991) Clinical use of ultrasound biomicroscopy. Ophthalmology, 98, 287-295.

[20] Turnbull, D.H., Starkoski, B.G., Harasiewicz, K.A., Semple, J.L., From, L., Gupta, A.K., Sauder, D.N. and Foster, F.S. (1995) A 40-100 MHz B-scan ultrasound backscatter microscope for skin imaging. Ultrasound in Medicine and Biology, 21, 79-88. doi:10.1016/0301-5629(94)00083-2

[21] Viren, T., Saarakkala, S., Kaleva, E., Nieminen, H.J., Jurvelin, J.S. and Toyras, J. (2009) Minimally invasive ultrasound method for intra-articular diagnostics of Cartilage degeneration. Ultrasound in Medicine and Biology, 35, 1546-1554. doi:10.1016/j.ultrasmedbio.2009.04.004

[22] Kurjak, A., Pooh, R.K., Merce, L.T., Carrera, J.M., Salihagic-Kadic, A. and Andonotopo, W. (2005) Structural and functional early human development assessed by three-dimensional and four-dimensional sonography. Fertility and Sterility, 84, 1285-1299. doi:10.1016/j.fertnstert.2005.03.084

[23] Passmann, C. and Ermert, H. (1996) A 100-MHz ultrasound imaging system for dermatologic and ophthalmologic diagnostics. IEEE Transactions on Ultrasonics Ferroelectrics and Frequency Control, 43, 545-552. doi: $10.1109 / 58.503714$

[24] Celermajer, D.S. et al. (1992) Noninvasive detection of endothelias dysfunction in children and adults at risk of atherosclerosis. Lancet, 340, 1111-1115. doi:10.1016/0140-6736(92)93147-F

[25] Vogt, M. and Ermert, H. (2005) Development and evaluation of a high-frequency ultrasound-based system for in vivo strain imaging of the skin. IEEE Transactions on Ultrasonics Ferroelectrics and Frequency Control, 52, 375-385. doi:10.1109/TUFFC.2005.1417260

[26] Cohn, N.A. et al. (1997) An elasticity microscope. 1. Methods. IEEE Transactions on Ultrasonics Ferroelectrics and Frequency Control, 44, 1304-1319. doi:10.1109/58.656634

[27] Liu, D. and Ebbini, E.S. (2008) Viscoelastic property measurement in thin tissue constructs using ultrasound. IEEE Transactions on Ultrasonics Ferroelectrics and Frequency Control, 55, 368-383. doi:10.1109/TUFFC.2008.655

[28] de Korte, C.L. et al. (2000) Characterization of plaque components with intravascular ultrasound elastography in human femoral and coronary arteries in vitro. Circulation, 102, 617-623.

[29] Liu, J. and Ferrari, M. (2003) A discrete model for the high frequency elastic wave examination on biological tissue. Computer Modeling in Engineering and Sciences, 4, 421-430.

[30] Liu, J. and Ferrari, M. (2002) Mechanical spectral signatures of malignant disease? A small-sample, comparative study of continuum vs nano-biomechanical data analysis. Disease Markers, 18, 175-183.

[31] Sakamoto, J.H. (2005) Molecular analysis of breast cancer utilizing tumor targeting ultrasound mechanical contrast agents. Thesis presented in partial fulfillment of the requirements for the Degree Doctor of Philosophy in the Graduate School of the Ohio State University, The Ohio State University, Columbus.

[32] Sakamoto, J.H., Gentile, F., Xie, B., Wang, L., Decuzzi, P., Rokhlin, S.I. and Ferrari, M. (2005) Nanomechanics and tissue pathology. In: Lee, A.P. and Lee, J.L., Eds., BioMEMS and Biomedical Nanotechnology, Vol. I: Prospectus, Biological and Biomedical Nanotechnology. Springer, New York.

[33] Böttger, H (1983) Principles of the theory of lattice dynamics, Physik-Verlag, Weinheim.

[34] Green, A.E. (1965) Micromaterials and multi-polar continuum mechanics. International Journal of Engineering Science, 3, 533-537. doi:10.1016/0020-7225(65)90033-9

[35] Edelen, D.G.B. (1976) Non local field theories. Continuum Physics, Academic Press, New York.

[36] Kunin, I.A. (1982) Elastic media with microstructure. I. One-dimensional models. Springer Series in Solid-State Sciences, Springer-Verlag, Berlin.

[37] Ferrari, M. (2000) Nanomechanics, and biomedical nanomechanics: Eshelby's inclusion and inhomogeneity problems at the discrete/continuum interface. Biomedical Microdevices, 2, 272-281.

[38] Ferrari, M., Granik, V.T., Imam, A. and Nadeau, J. (1997) Advances in doublet mechanics. Springer, Heidelberg.

[39] Granik, V.T. and Ferrari, M. (1993) Microstructural mechanics of granular materials. Mechanics of Materials, 15, 301-322. doi:10.1016/0167-6636(93)90005-C

[40] Sakamoto, J.H., Smith, B.R., Xie, B., Rokhlin, S.I., Lee, S.C. and Ferrari, M. (2005) The molecular analysis of breast cancer utilizing targeted nanoparticle based ultrasound contrast agents. Technology in Cancer Research \& Treatment, 4, 627-636.

[41] Williams, A. (2003) Transdermal and topical drug delivery: From theory to clinical practice. Pharmaceutical Press, London.

[42] Zong, X.L., Laine, A.F. and Geiser, E.A. (1998) Speckle reduction and contrast enhancement of echocardiograms via multiscale nonlinear processing. IEEE Transactions on Medical Imaging, 17, 532-540. doi:10.1109/42.730398

[43] Lehmann, M. (1998) Statistical theory of two-wave speckle interferometry and its application to the optimization of deformation measurements. Thèse Ecole Polytechnique Fédérale de Lausanne EPFL, No. 1797. 


\section{APPENDIX A}

The propagation of the waves in the media can be unambiguously identified specifying thirteen angles (Figure 3)

$$
\begin{aligned}
& \theta_{0}=\theta_{1}, \theta_{5}=\theta_{3}, \theta_{6}=\theta_{4}, \theta_{9}=\theta_{7}, \\
& \theta_{10}=\theta_{8}, \theta_{11}=\theta_{0}, \theta_{12}=\theta_{2}
\end{aligned}
$$

where the identities in (9) stem from the second law of optics of reflection. Given the mechanical properties of the media and $\theta_{0}$, Snell's law may be used to derive six further independent equations having the form

$$
\frac{\sin \theta_{i}}{\sin \theta_{t}}=\frac{c_{t}}{c_{i}}
$$

where $\theta_{i}$ and $\theta_{t}$ are the incident and transmitted wave directions, and the $c_{i}$ the wave phase velocities. Using Equations (9) and (10) it is thus straightforward expressing any characteristic parameter $\theta_{i}$ in terms of the sole incident angle $\theta_{0}$. The displacements $\boldsymbol{u}^{(n)}$ can be expressed as

$$
\begin{aligned}
& \boldsymbol{u}^{(n)}=A_{n} \boldsymbol{d}^{(n)} \exp \left(i \xi^{(n)}\right) ; \\
& \xi^{(n)}=k_{n}\left(\boldsymbol{x} \cdot \boldsymbol{p}-c_{n} t\right)
\end{aligned}
$$

with (n) an index indicating the (n)th wave, $A_{n}$ the amplitude of the waves, $\boldsymbol{x}$ is the position vector, $\boldsymbol{d}^{(n)}$ the particle motion unit vector, $\boldsymbol{p}$ is propagation unit vector and $k_{n}=2 \pi / \lambda$ the wave number.

The most relevant coefficient in the system is the reflection coefficient $R_{1}=A_{1} / A_{0}$ which can be determined imposing at each interface suitable boundary conditions for either the displacements and the stresses

$$
\begin{aligned}
& \sum_{i=1}^{m} u_{1}^{(i)}, \sum_{i=1}^{m} u_{2}^{(i)}, \\
& \sum_{i=1}^{m} \sigma_{22}^{(i)}, \sum_{i=1}^{m} \sigma_{12}^{(i)}
\end{aligned}
$$

here, $m$ is the number of perturbations insisting upon the same boundary, $u_{1}, u_{2}, \sigma_{22}$ and $\sigma_{12}$ are the displacements and the stresses, of tangential and normal type respecttively. Relations (12) generate a total of twelve equations meaning that the problem of solving for the twelve reflection coefficients (four per interface) is completely determined.

The reflection coefficients $R_{i}$ depend upon the waves amplitude $A_{n}$ and thus, through Equation (11), also upon the displacements $\boldsymbol{u}^{(n)}$. On the other hand, displacements may be conveniently rephrased in terms of stresses, provided that appropriate constitutive equations are used. The waves (0), (1), (2), (3), (4), (5), (6), (11) and (12) can be handled using the conventional theory of elasticity in that travel in continuum media. On the contrary DM is necessary in describing waves (7), (8), (9) and (10) which propagate in the biological, non continuum substrate. In this perspective, $M$ has been chosen as $M=2$, that is the smallest value of $M$ that retains the scale features of DM. It is assumed that the dynamic process is isothermal and the volume forces vanish. The particle interactions are assumed to be longitudinal (central), so that the shear and torsion microstresses vanish everywhere in the body. Recalling (4), for $M=2$ the elongation microstrains are derived as

$$
\varepsilon_{\alpha}=\tau_{\alpha i} \tau_{\alpha j} u_{i, j}+\frac{1}{2} \tau_{\alpha i} \tau_{\alpha j} \tau_{\alpha k} u_{i, j k}
$$

while, recalling (5), the micro moduli matrix $A_{\alpha \beta}$ takes the form

$$
\begin{gathered}
A_{\alpha \beta}=\left(\begin{array}{cccccc}
A_{11} & A_{12} & A_{12} & 0 & A_{15} & A_{15} \\
& A_{11} & A_{12} & A_{15} & 0 & A_{15} \\
& & A_{11} & A_{15} & A_{15} & 0 \\
& & & A_{44} & 0 & 0 \\
& & & & A_{44} & 0 \\
\text { Sym } & & & & & A_{44}
\end{array}\right) \\
\qquad=A_{11}-A_{44} ; \mu=\frac{1}{4} A_{44}
\end{gathered}
$$

where solely the two constants $A_{11}$ and $A_{44}$ are independent. Under these conditions, relation (8) is simplified into

$$
\sigma_{i j}=\sum_{\alpha=1}^{n}\left(\tau_{\alpha i} \tau_{\alpha j} p_{\alpha}-\frac{1}{2} \eta_{\alpha} \tau_{\alpha i} \tau_{\alpha j} \tau_{\alpha k} p_{\alpha, k}\right)
$$

The unit vectors $\tau$ are chosen as follows

$$
\begin{aligned}
& \tau_{1}=\left(\begin{array}{l}
1 \\
0 \\
0
\end{array}\right), \tau_{2}=\left(\begin{array}{l}
0 \\
1 \\
0
\end{array}\right), \tau_{3}=\left(\begin{array}{l}
0 \\
0 \\
1
\end{array}\right), \\
& 2 \tau_{4}=\left(\begin{array}{c}
0 \\
\sqrt{2} \\
\sqrt{2}
\end{array}\right), 2 \tau_{5}=\left(\begin{array}{c}
\sqrt{2} \\
0 \\
\sqrt{2}
\end{array}\right), 2 \tau_{6}=\left(\begin{array}{c}
\sqrt{2} \\
\sqrt{2} \\
0
\end{array}\right)
\end{aligned}
$$

and correspondingly the doublets distribute in space as in Figure 13. Notice that the problem is fully three-dimensional.

Substituting back within relations (12) the stresses and displacements written in terms of the amplitudes $A_{n}$ according to Equation (11) and Equations from (13) to (16), a subset of 12 explicit equations is derived, which may be clothed in matrix form as to obtain $\boldsymbol{M} \cdot \boldsymbol{R}=\boldsymbol{B}$, where $\boldsymbol{M}$ is the $[12 \times 12]$ coefficients matrix, $\boldsymbol{B}$ the $[12 \times 1]$ vector comprising the known terms and $\boldsymbol{R}$ represents the $[12 \times 1]$ vector enclosing of the unknown reflection coefficients $R_{i}$. Both $\boldsymbol{M}$ and $\boldsymbol{B}$ depend upon a number of 


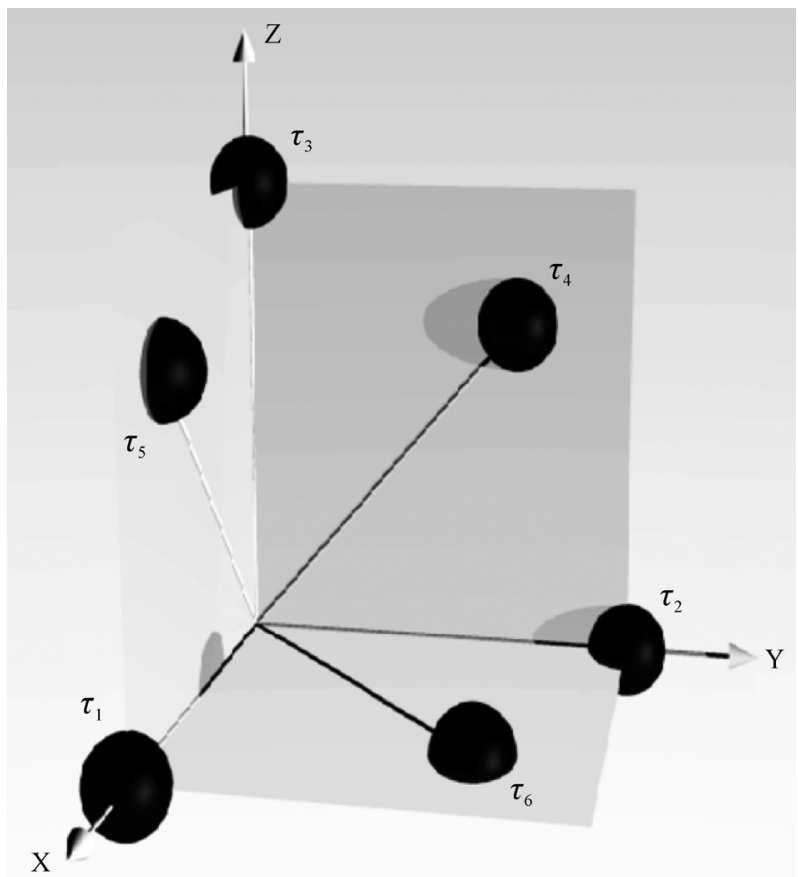

Figure 13. The doublets distributing in space as to form a 3D arrangement.

parameters, namely: the micro mechanical properties of the biological substrate (density, nodal separation distance $\eta_{\alpha}$, micro moduli $A_{\alpha \beta}$ ); the classical continuum mechanics properties of the glass substrates and of the auxiliary layer (density, constants of Lamè $\lambda$ and $\mu$ ); the thickness of the layers $\left(h_{1}, h_{2}\right)$; the initial set-up angle $\theta_{0}$; the phase velocities $c_{i}$; the time harmonic waves' angular frequency $\omega=2 \pi f$. Noticeably, while conventionally the relation between $\omega$ and $k_{i}$ is represented by the celebrated relation $k_{i}=\omega / c_{i}\left(c_{i}=(E / \rho)^{1 / 2}\right)$ in the framework of DM it assumes a far more elaborated form, for either pressure $(\mathrm{P})$ or shear $(\mathrm{S})$ waves

$$
\begin{aligned}
-\rho \omega^{2}= & -A_{44}\left(A_{11}-\frac{1}{2} A_{44}\right) k^{2} \\
& +\frac{1}{4} \eta^{2}\left(A_{11}-\frac{\sqrt{2}}{2} A_{44}+\frac{1}{4} A_{44}\right) k^{4},(P) \\
-\rho \omega^{2}= & -\frac{1}{4} A_{44} k^{2}+\eta^{2} A_{44} \frac{k^{4}}{32},(S)
\end{aligned}
$$

and we shall call the latter equations of dispersion in that demonstrate that propagation in discrete-media is dispersive, and strongly influenced by the micro moduli and by the scale length.

\section{APPENDIX B}

In its present form, the model presented in the paper does not take into account diffraction or scattering. Coupling these effects with the DM theory would require considerable mathematical efforts which are considered outside the scope of this study and left for future investtigations. While there are several practical ways to reduce speckle (such as the use of spatial and frequency compounding techniques [42], it is important to understand that, under certain circumstances a simple reflection/refraction model is still sufficient for describing tissue/US interaction.

A practical proof for this statement is the fact that ultrasound reflection techniques are commonly employed to accurately measure the thickness of different tissues or tissue layers and for non destructive testing of materials. These circumstances are insonicating from different angles and averaging the results. This sentence is further substantiated in the following.

A speckle field arises when a wave impinges on a rough surface, generating a scattered wave radiating in all directions. Each echo signal is the combination of the many signals coming from a group of scatterers within the resolution cell. At each point, the amplitude of the echo signal depends on whether constructive or destructtive interference predominates. Since the scattered wave emanates from numerous contributors, it is appropriate to characterize it in statistical terms. If this field is integrated over a finite detector area (that would be the US receiver) the probability distribution of the integrated intensity $I$ is [43]

$$
p_{I}(I)=\left(\frac{m}{\langle I\rangle}\right)^{m} \frac{I^{m-1}}{\Gamma(m)} \exp \left(-\frac{m}{\langle I\rangle} I\right)
$$

where $\Gamma(m)$ is the Gamma function, the operator $<\cdot>$ stands for spatial average and thus $\langle I\rangle$ would correspond to the "true" mean intensity in the limit of a perfectly flat reflective surface, and $m$ is, to a first approximation, the number of correlation cells or speckles falling onto the detection area. Figure 14 illustrates Equation (18) for different $m$ 's. Notice that for increasing $m$

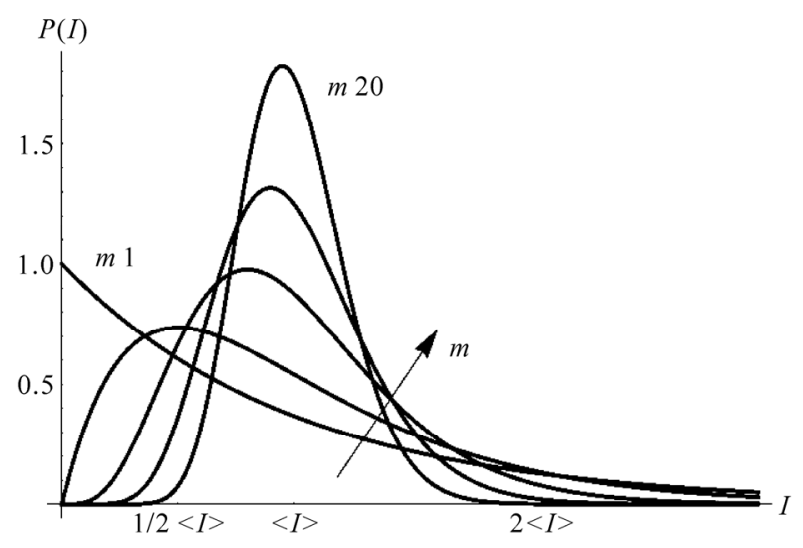

Figure 14. The probability distribution of the speckle integrated intensity $I$ for different $m$ 's. 
the diagrams turn into quasi-Gaussian with most probable intensity $\langle I\rangle$. Equivalently, in the limit of large detector area, the speckles would just represent a background noise that would have negligible influence on the response of the sample. Founding on these considerations, scattering effects can be reasonably neglected.
Note that by large detector area we simply mean an area containing a large number of scatterers compared to the resolution cell, which is equivalent to state that the tissue scatterer density satisfies the requirement for obtaining fully developed Raleigh backscatterers and thus $m \gg 1$. 